\title{
ANÁLISE DA APLICAÇÃO DO DELINEAMENTO COMPOSTO CENTRAL EM ENSAIOS DE TRATABILIDADE DA ÁGUA
}

\section{ANALYSIS OF THE APPLICATION OF THE CENTRAL COMPOSITE DESIGN ON WATER TREATABILITY TESTS}

\author{
B. R. V. SIMÕES ${ }^{1}$, D. S. GOULART ${ }^{1}$, M. P. M. COMBATT ${ }^{1}$, J. F. P. PIMENTA ${ }^{1}$, M. E. L. R. QUEIROZ ${ }^{1}$ \\ e D. J. SILVA ${ }^{1, *}$
}

${ }^{1}$ Federal University of Viçosa, Department of Chemistry, Viçosa, Minas Gerais, Brazil

${ }^{*}$ Corresponding author. Federal University of Viçosa, Department of Chemistry, Viçosa, Minas Gerais, Brasil, Phone: +55 31984498989

e-mail address: deusanilde.silva@gmail.com (D. J. Silva).

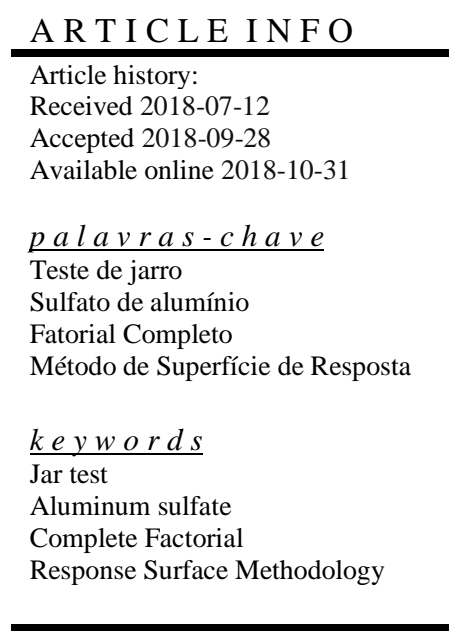

\begin{abstract}
A B S T R A C T
Due to the periods of water crisis in the different Regions of Brazil it is necessary to use new sources of capitation and treatability trials are indispensable. The feasibility of using the Central Compound Design (DCC) in the water treatability trials as a substitution for the classical analysis of the coagulation diagram, by complete factorial, was studied. The parameters of interest were the aluminum sulphate dose (5 and $\left.60 \mathrm{mg} \cdot \mathrm{L}^{-1}\right)$ and initial $\mathrm{pH}(6$ and 8 ). The response variable was the turbidity removal efficiency. It was possible to maximize the response at the initial $\mathrm{pH}$ on point of 7.23 and the dosage of $49.9 \mathrm{mg} \mathrm{L}^{-1}$ and found regions of removal greater than $80 \%$ common to both methods, indicating that DCC may be a viable tool for analysis as compared to the conventional method, having the advantage of reducing the number of tests and, consequently, the operating time.
\end{abstract}

\section{R E S UM O / R E S U M E N}

Devido aos períodos de crise hídrica nas diferentes Regiões do Brasil é necessário utilizar novas fontes de captação, sendo indispensáveis ensaios de tratabilidade. Foi estudada a viabilidade do uso do Delineamento Composto Central (DCC) nos ensaios de tratabilidade de água como substituição à análise clássica do diagrama de coagulação, por fatorial completo. Os parâmetros de interesse foram a dose de sulfato de alumínio $\left(5\right.$ e $\left.60 \mathrm{mg} \mathrm{L}^{-1}\right) e$ o pH inicial (6 e 8). A variável resposta foi a eficiência de remoção de turbidez. Foi possível maximizar a resposta no ponto de $\mathrm{pH}$ inicial de 7,23 e dosagem de 49,9 $\mathrm{mg} \mathrm{L}^{-1}$ e encontrar regiões de remoção superior a $80 \%$ comuns a ambos os métodos, indicando que o DCC pode ser uma ferramenta viável para análise se comparada com o método convencional, tendo como vantagem a redução da quantidade de ensaios e, consequentemente, o tempo de operação. 


\section{INTRODUÇÃO}

Existem diversas tecnologias de tratamento de água, a depender da qualidade da água bruta utilizada, que podem ser resumidas em dois grupos, sem coagulação química e com coagulação química, e precedidas ou não por um pré-tratamento, segundo Di Bernardo et al. (2005). Na maioria dos estados brasileiros, de acordo com dados do IBGE (2008), o tipo de tratamento de água utilizado é o convencional que consiste em um processo com etapas subsequentes: coagulação/floculação, decantação, filtração e desinfecção.

Cada etapa do tratamento é de grande importância para garantir a qualidade da água de abastecimento para consumo humano, por isso deve haver um controle e supervisão dos parâmetros da água em diversas etapas do tratamento para que todas as exigências da PRC $\mathrm{n}^{\circ}$ 5, 28 de set. 2017, anexo XX, do Ministério da Saúde, sejam atendidas.

Di Bernardo et al. (2005) afirma que, do ponto de vista tecnológico, água de qualquer qualidade pode ser, teoricamente, transformada em água potável, porém, os custos envolvidos e a operação podem inviabilizar o uso de um determinado curso de água como fonte de abastecimento. Dessa forma, se faz necessário buscar alternativas tecnológicas compatíveis com a qualidade da água do manancial sendo que, somente com a realização de análises laboratoriais e com a execução de ensaios em bancada ou instalações de unidades piloto, é que algumas tecnologias podem ser consideradas e outras descartadas.

A água utilizada no presente estudo provém da microbacia do Córrego dos Araújos, que compõe a malha hídrica da Universidade Federal de Viçosa (UFV), campus Viçosa. Análises periódicas são realizadas pela Estação de Tratamento de Água da UFV (ETA-UFV), indicando elevado nível de eutrofização, processo que, segundo Smith et al. (2009), provoca o crescimento excessivo de plantas devido ao enriquecimento de nutrientes causado pela atividade humana e é o principal problema enfrentado atualmente pela maioria das águas superficiais.

A escolha desse manancial para o estudo foi motivada pelo superávit hídrico que o Córrego dos Araújos apresenta, caracterizando-o como uma boa alternativa de oferta complementar de água. Tem-se que, de acordo com estudo realizados por Gomes et al. (2015) com dados do Instituto Mineiro de Gestão das Águas (IGAM), seu valor de $\mathrm{Q}_{7,10}$ é de 44,64 $\mathrm{L} \mathrm{s}^{-1}$, representando uma considerável vazão mínima de sete dias de duração em dez anos de recorrência. Devido aos períodos de crise hídrica enfrentados nos últimos anos, é de grande relevância verificar a possibilidade de utilizar novas fontes de captação para futuro abastecimento público, sendo necessária a realização de ensaios com intuito de averiguar a tratabilidade da água e a viabilidade econômica, bem como definir a faixa de coagulante adequada para o tratamento.

Quando há necessidade de adequar/otimizar um tratamento já existente, ou ainda projetar uma nova ETA, usualmente são construídos diagramas de coagulação, pois, como relatado por Ferrari et al. (2012), a depender da natureza da água e das propriedades dos colóides nela presentes, o desempenho da coagulação poderá variar consideravelmente. Para tal, são realizados testes de jarros com diferentes combinações de dosagem do coagulante e de valores de $\mathrm{pH}$, abrangendo ampla região de estudo. Bartiko et al. (2015) e
Morais et al. (2012) descrevem que, a partir dos resultados obtidos, são criadas curvas de isovalor do parâmetro avaliado, normalmente turbidez e cor remanescentes, sendo possível definir algumas regiões onde determinados mecanismos de coagulação são predominantes.

Libanio (2010) define que existem quatro tipos de mecanismos de coagulação, os quais são: adsorção e neutralização de cargas; varredura; adsorção e formação de pontes interparticulares e compressão da camada difusa. Quando o coagulante escolhido é um sal inorgânico, os mecanismos predominantes são o de varredura e o de adsorção e neutralização de cargas, onde o primeiro é o mais utilizado nas ETA's, pois os flocos formados são maiores e sedimentam-se com maior facilidade, ocorrendo em valores mais elevados de $\mathrm{pH}$ e de doses de coagulante, o qual precipita como hidróxido metálico. Já o segundo é mais utilizado quando se emprega a filtração direta, não sendo necessária a formação de flocos para posterior sedimentação e ocorre em valores de $\mathrm{pH}$ mais baixos, com o coagulante apresentando-se em sua forma cationica, segundo Duan et al. (2003) e Pavanelli et al. (2001).

Para avaliação de dois fatores de interesse, tais como dose de coagulante e pH, de acordo com Ribeiro Júnior (2011), pode-se planejar experimentos por meio da combinação dos seus níveis escolhidos, comumente empregado na construção dos diagramas de coagulação, ou ainda por um Delineamento Composto Central (DCC), o que possibilita, através de análise estatística, obter conclusões por meio de superfícies de resposta, como citado por Bezerra (2008). Uma das principais diferenças entre os métodos está na quantidade de ensaios necessários, uma vez que o Fatorial Completo é mais extenso.

O presente trabalho objetivou a análise de viabilidade de utilização do Delineamento Composto Central frente ao Fatorial Completo, visando máxima remoção de turbidez. Ainda, é proposta a avaliação da tratabilidade da água do Córrego dos Araújos por parte das etapas do tratamento convencional em escala de bancada.

\section{MATERIAL E MÉTODOS}

\subsection{Coleta e caracterização da água}

O experimento foi conduzido na ETA-UFV, campus Viçosa, sendo a água do Córrego dos Araújos captada no dia 28 de maio de 2018. Para tal, foram utilizados recipientes plásticos com capacidade de vinte litros, posteriormente armazenada em tanques de mesmo material, com capacidade de cem litros e homogeneizada imediatamente antes da sua utilização.

A caracterização da água de estudo foi realizada avaliando o pH, quantificado com o auxílio de um pHmetro portátil Digmed DM-2P; a turbidez, com o auxílio de um turbidímetro HACH 2100AN; e a cor aparente, com o emprego de um fotocolorímetro Policontrol Aquacolor. Todas essas análises, bem como as realizadas após os ensaios, seguiram metodologias descritas no Standard Methods For the Examination of Water and Wastewater por Eaton (2017).

\subsection{Teste de jarros}

As etapas de coagulação/floculação e sedimentação foram realizadas em dois agitadores Jar Tests, Nova Ética Modelo 218 LDB, contendo seis jarros com capacidade de dois litros cada, seguindo a metodologia proposta por Di Bernardo et al. (2011). Os parâmetros hidráulicos utilizados foram os 
mesmos da ETA-UFV para uma vazão de $40 \mathrm{~L} \mathrm{~s}^{-1}$ e estão expressos na Tabela 1 , onde $\mathrm{G}$ é o gradiente de velocidade, $\left[\mathrm{s}^{-1}\right]$. Na última etapa, para decantação dos flocos, utilizou-se uma velocidade de sedimentação de $1,4 \mathrm{~cm} \mathrm{~min}^{-1}$.

Tabela 1 - Parâmetros hidráulicos para os testes de jarros.

\section{Parâmetro}

Mistura Rápida

Floculação

Sedimentação

O coagulante utilizado foi o sulfato de alumínio $50 \%$ $(\mathrm{m} / \mathrm{v})$ líquido, isento de ferro, com teor de $\mathrm{Al}_{2} \mathrm{O}_{3}$ de $8,12 \%$, que foi diluído para se obter uma solução de $250 \mathrm{~mL}$ a $2 \%(\mathrm{~m} / \mathrm{v})$, que é a concentração utilizada pela ETA-UFV. Os valores de $\mathrm{pH}$ foram ajustados com auxílio de conta-gotas utilizando o agente acidificante ácido sulfúrico $\left(1,0 \mathrm{~mol} \mathrm{~L}^{-1}\right)$ ou o alcalinizante

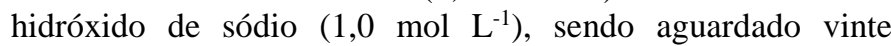
segundos para a estabilização. A temperatura da água de estudo foi de $20 \pm 1^{\circ} \mathrm{C}$.

Os ensaios em equipamento Jar Test iniciaram-se com o preenchimento dos jarros utilizando dois litros da água de estudo, com os valores já ajustados de $\mathrm{pH}$. No início da mistura rápida, as doses definidas de coagulante foram adicionadas aos jarros. Após os ensaios, foram retiradas amostras de cada jarro para análises de turbidez remanescente. Com base nesses dados obtidos, foram calculadas as taxas de remoção pela Equação 1.

$\mathrm{T}(\%)=\frac{\mathrm{T}_{\mathrm{i}}-\mathrm{T}_{\mathrm{f}}}{\mathrm{T}_{\mathrm{i}}} \times 100$ onde, T é remoção de turbidez [\%], $\mathrm{T}_{\mathrm{i}}$ é turbidez inicial [uT] e $\mathrm{T}_{\mathrm{f}}$ é turbidez final [uT].

\subsection{Planejamento experimental}

Para os ensaios, foram escolhidos dois fatores de interesse, $\mathrm{pH}$ inicial e dose de coagulante $\left(\mathrm{mg} \mathrm{L}^{-1}\right)$, combinados em níveis de modo aleatório. Esses parâmetros são os utilizados diariamente nos ensaios de tratabilidade nas ETA's, uma vez que determinadas combinações entre eles podem conduzir a uma melhor eficiência de coagulação, segundo Bartiko et al. (2015).

Para o $\mathrm{pH}$ inicial da água bruta, foi definida uma faixa de 6 a 8 e para a dosagem do coagulante, 5 a $60 \mathrm{mg} \mathrm{L}^{-1}$. A escolha dessas faixas baseou-se em diagramas de coagulação para sulfato de alumínio, como o representado na Figura 1, que relaciona o $\mathrm{pH}$ da mistura com a dosagem e delimita as regiões dos diferentes mecanismos de coagulação. A preferência por esses valores teve como principal objetivo cingir o mecanismo de varredura, citado por Amirtharajh (1989), intensivamente empregado em estações de tratamento convencional de água.

É importante ressaltar que este diagrama foi utilizado somente como um guia, pois sabe-se que o tipo de sulfato de alumínio para sua construção é diferente do utilizado nesse trabalho. Além disso, a abscissa da Figura 1 representa o $\mathrm{pH}$ da mistura durante a coagulação e, devido a dificuldades encontradas para medir e controlar esse tipo de valor de $\mathrm{pH}$, foi preferível utilizar o $\mathrm{pH}$ inicial como a variável de entrada.

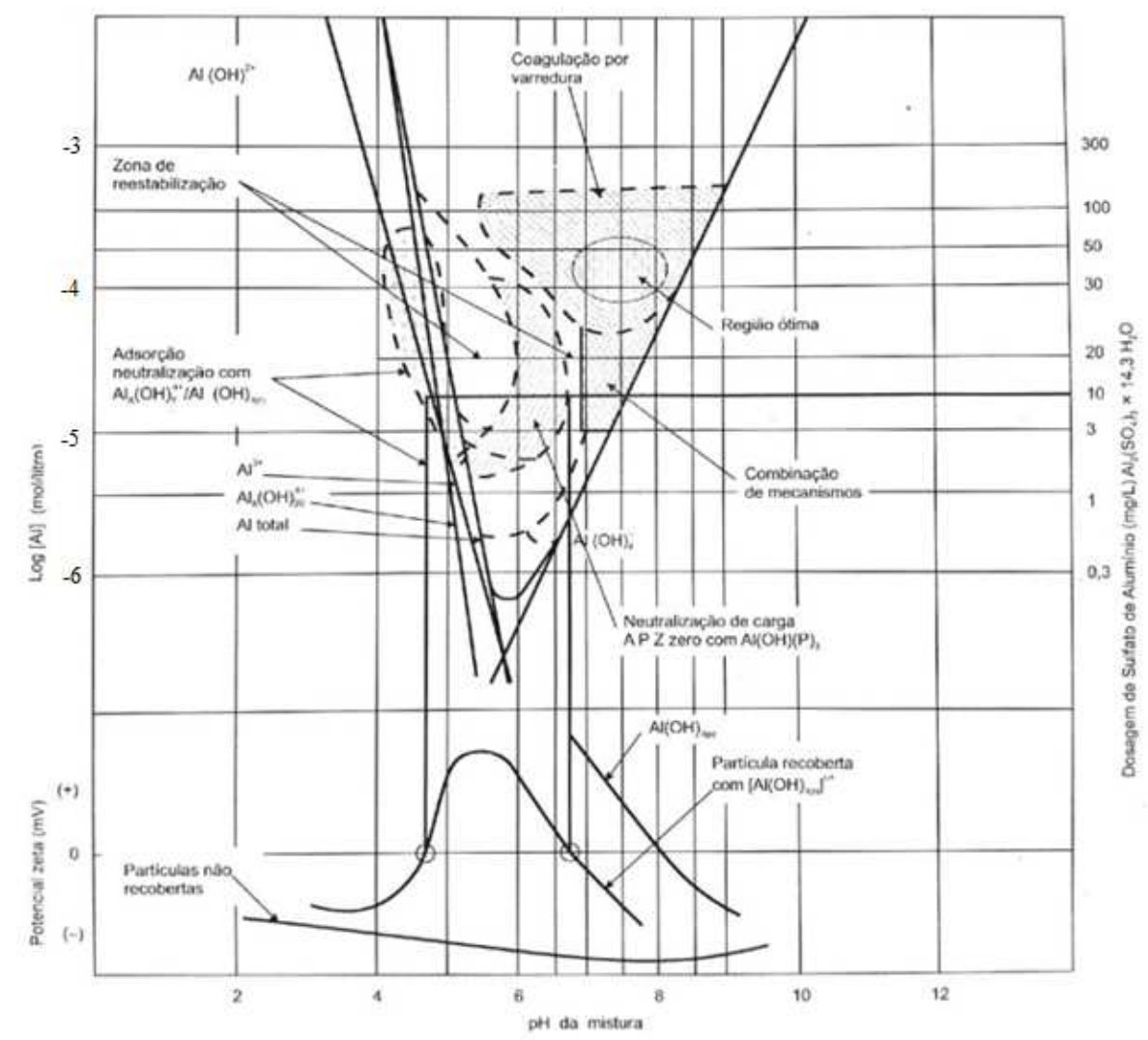

Figura 1 - Diagrama típico de coagulação do sulfato de alumínio. Fonte: Amirtharajh (1989). 
Os planejamentos experimentais foram realizados com auxílio do software MINITAB® 17 (Minitab Inc., USA). Nos ensaios com Jar Test para o Fatorial Completo, definiu-se cinco níveis para o $\mathrm{pH}(6,0 ; 6,5 ; 7,0 ; 7,5 ; 8,0)$ e doze níveis para a dosagem $(5 ; 10 ; 15 ; 20 ; 25 ; 30 ; 35 ; 40 ; 45 ; 50 ; 55 ; 60)$ que, combinados, resultaram em sessenta ensaios.

Já para o Delineamento Composto Central, foi utilizado um Método de Superfície de Resposta (MSR) com o delineamento estatístico instalado sob o Delineamento Inteiramente Casualizado (DIC), com os ensaios expressos na Tabela 2, onde pHi é pH inicial e S é dose em $\mathrm{mg} \mathrm{L}^{-1}$.

Tabela 2 - Planejamento experimental para o DCC.

\begin{tabular}{ccc} 
Ensaio & $\mathrm{pHi}$ & $\mathrm{S} / \mathrm{mg} \mathrm{L}^{-1}$ \\
1 & 8 & 5 \\
2 & 8 & 60 \\
3 & 6 & 5 \\
4 & 6 & 60 \\
5 & 7 & 32,5 \\
6 & 7 & 71,4 \\
7 & 7 & 32,5 \\
8 & 5,59 & 32,5 \\
9 & 7 & 32,5 \\
10 & 7 & 32,5 \\
11 & 7 & 0,1 \\
12 & 8,41 & 32,5 \\
13 & 7 & 32,5 \\
\hline
\end{tabular}

Foram definidos nove tratamentos, a partir dos mesmos limites inferior e superior, representados por quatro pontos cúbicos, quatro pontos axiais e um ponto central com quatro repetições $\mathrm{O}$ alfa escolhido, que determina a distância entre pontos experimentais, foi o padrão, com seu valor maior que 1 , posicionando os pontos axiais além dos limites dos pontos cúbicos, como mostra a Figura 2.

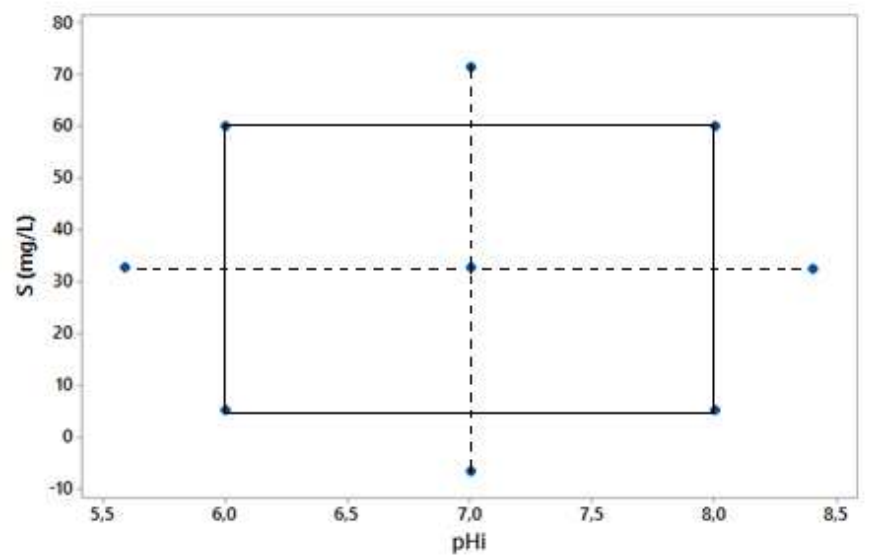

Figura 2 - Gráfico de dispersão dos pontos experimentais da dose de coagulante (S) e do Ph inicial (pHi), com valor de alfa padrão.
A partir dos dados obtidos, foi realizada uma análise da variável reposta, assim como Combatt et al. (2017), onde valor p representa a probabilidade de significância dos termos e o nível de confiança foi de $95 \%$, ou seja, um nível de significância de 0,05 .

\subsection{Análise econômica}

A análise de viabilidade econômica fundamentou-se nos custos envolvidos com os operadores levando em consideração o tempo necessário para execução dos ensaios.

É possível realizar seis ensaios simultâneos em um mesmo Jar Test, dessa forma, com os níveis estabelecidos para as variáveis de interesse, foram necessárias três corridas para o DCC e dez corridas para as combinações por fatorial. A partir da Tabela 1, sabe-se que a duração de cada corrida foi de 54 min, sendo necessários, aproximadamente, $60 \mathrm{~min}$ adicionais para o preparo das dosagens, análises das amostras e limpeza dos jarros.

\section{RESULTADOS E DISCUSSÕES}

A partir de análises realizadas, foram encontrados valores médios de $\mathrm{pH}$, de turbidez e de cor aparente para caracterização da água de estudo. Esses resultados são apresentados na Tabela 3 , juntamente com valores de análises da água utilizada pela ETA-UFV, realizadas no dia 28 de maio de 2018 .

Tabela 2 - Média dos parâmetros da água bruta de estudo e da água bruta utilizada na ETA-UFV.

\begin{tabular}{ccc} 
& & Água ETA- \\
Parâmetro & Água de Estudo & UFV \\
\hline pH & 7,04 & 7,74 \\
Turbidez / uT & 33,13 & 10,49 \\
Cor aparente / Uh & 121,87 & 26,39 \\
\hline
\end{tabular}

Foi possível perceber que a cor aparente e a turbidez da água bruta de estudo são superiores aos valores encontrados para a água bruta utilizada no tratamento da ETA-UFV.

Depois de finalizados os ensaios, para a análise dos resultados da água de estudo, foram calculados as eficiências de remoção de turbidez e de cor por meio da Equação 1. Entretanto, de modo a facilitar a comparação dos métodos estatísticos, este trabalho apresentou somente a análise realizada de remoção de turbidez. Esses valores foram inseridos no software de modo a ser possível a análise comparativa dos dois métodos de estudo.

Dentre todos os ensaios realizados, a maior eficiência de remoção obtida foi de $89,81 \%$, em um valor de pHi igual a 7 e dosagem de $32,5 \mathrm{mg} \mathrm{L}^{-1}$, que resultou em um valor de turbidez remanescente de 3,23 uT, após a sedimentação. Segundo estudos realizados por Oliveira (2009) na ETA-UFV, 75\% dos dados de água filtrada com turbidez inferior a 0,5 uT, limite exigido pela PRC n 5, 28 de set. 2017, anexo XX, estão relacionados com turbidez decantada abaixo de 4,0 uT. Dessa forma, entende-se que a água de estudo pode atender às exigências de valores de turbidez após o tratamento convencional completo para o abastecimento público. 


\subsection{Fatorial Completo}

Esse método foi baseado em sessenta ensaios e pode-se perceber que suas combinações percorrem toda a faixa dentro dos limites estabelecidos de forma que o $\mathrm{pH}$ varia seu valor em intervalos de 0,5 e a dose a cada $5 \mathrm{mg} \mathrm{L}^{-1}$. Depois de realizadas as análises, foi possível obter o gráfico de contorno apresentado na Figura 3, o qual apresenta regiões de percentual de remoção da turbidez em relação ao pHi e à dosagem utilizados.

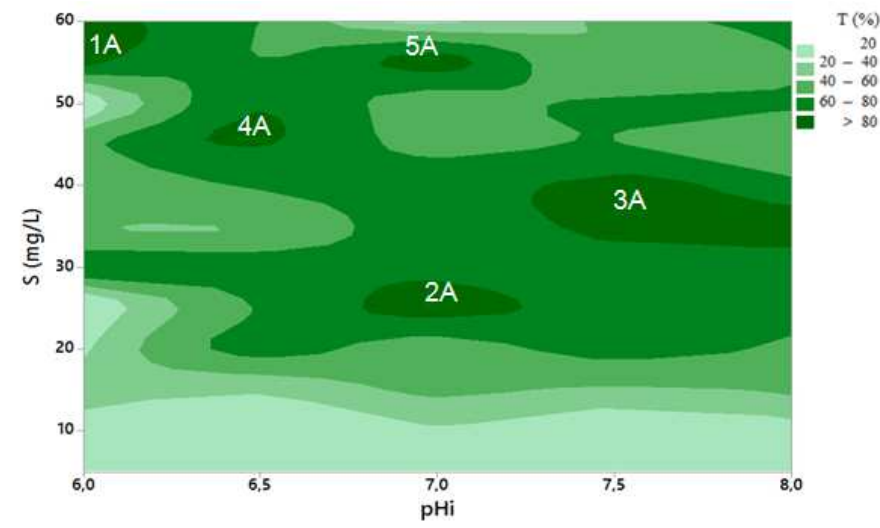

Figura 3 - Gráfico de contorno da remoção de turbidez $\mathbf{T}(\%)$ versus S; pHi para os ensaios pelo Fatorial Completo.

Nele, pode-se observar o surgimento de cinco regiões mais escuras onde a remoção de turbidez foi superior a $80 \%$. Algumas delas apresentam uma dilatação ao longo do eixo das abscissas, que se pode inferir que o pHi tem uma menor influência, em relação à dose de sulfato de alumínio, na remoção de turbidez.

Pode-se perceber que a Região 2A seria a mais adequada para a operação de uma ETA que utilizasse uma água bruta semelhante à de estudo, com o pH inicial próximo de 7, pois dessa forma não haveriam custos em relação ao ajuste do $\mathrm{pH}$. Entretanto, a Região 3A, devido à sua maior área, apresenta-se como uma boa alternativa para uma operação com água bruta com pH inicial maior que 7. Representa uma área de segurança onde variações no valor de $\mathrm{pH}$ inicial e na dose de coagulante não influenciam de forma significativa a eficiência de remoção de turbidez.

\subsection{Delineamento Composto Central}

A partir dos valores de remoção de turbidez obtidos pelos tratamentos do DCC, obteve-se o gráfico de contorno dos dados reais, indicado na Figura 4. É possível observar três regiões mais escuras bem definidas cuja remoção dá-se em valores superiores a $80 \%$. A região $1 \mathrm{~B}$ localiza-se próxima ao limite superior da dose estabelecida (60 $\mathrm{mg} \mathrm{L}^{-1}$ ) e ao limite inferior de $\mathrm{pHi}$, em torno de 6. Percebe-se que ela se encontra em uma área coincidente com a região $1 \mathrm{~A}$ do gráfico de contorno dos ensaios realizados pela combinação Fatorial, Figura 3. Além disso, a região $2 \mathrm{~B}$ também se encontra em valores semelhantes aos da região $2 \mathrm{~A}$, com $\mathrm{pH}$ em torno de 7 e dose próxima de $30 \mathrm{mg} \mathrm{L}^{-1}$.

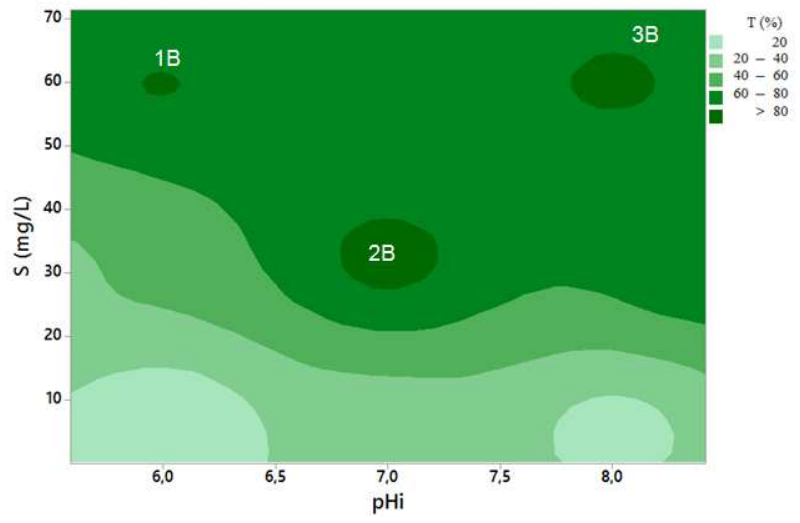

\section{Figura 4 - Gráfico de contorno de remoção de turbidez T(\%) versus S; pHi para os ensaios do Delineamento Composto Central.}

Pode-se notar que no Fatorial Completo não há extrapolações dos limites inferior e superior definidos pelos níveis de pHi e dose de coagulante para ambos os métodos. Dessa forma, como não houve ensaios com valores de $\mathrm{pHi}$ maiores que 8 e concentração maiores que $60 \mathrm{mg} \mathrm{L}^{-1}$, o gráfico de contorno não mostrou regiões acima desse valor. Por isso, a região $3 \mathrm{~B}$, com remoção de turbidez maior que $80 \%$ mostrada pelo método DCC, não foi contemplada na Figura 3. Além disso, as Regiões 3A, 4A e 5A da Figura 3, foram negligenciadas pelo gráfico de contorno da Figura 4.

Ao analisar o diagrama da Figura 1, pode-se perceber que existem regiões onde certos mecanismos de coagulação predominam. Isso explica o surgimento de regiões, nas Figuras 3 e 4, com elevada eficiência de remoção de turbidez em diferentes pares dos parâmetros de interesse.

Para ajuste do modelo de máxima remoção de turbidez em função do $\mathrm{pH}$ inicial e da dose de sulfato de alumínio, foram considerados somente os termos significativos, considerando o nível de confiabilidade de 95\%. A Figura 5 apresenta o gráfico de pareto dos efeitos padronizados e mostra que a interação entre os fatores $\mathrm{pHi}$ e dose $\left(\mathrm{pHi}^{*} \mathrm{~S}\right)$ foi o único termo não significativo, pois apresenta um valor inferior à linha de referência de 2,45. Com isso, não foi possível concluir que há uma associação estatisticamente significativa entre a variável de resposta e essa interação. Isso pode ser explicado pelo fato da variável de entrada utilizada ter sido o pH inicial da água, e não o pH de coagulação. Além disso, como o gráfico de paretos exibe o valor absoluto dos efeitos, observa-se também que a dose $(\mathrm{S})$ apresenta uma maior magnitude em relação ao pHi.

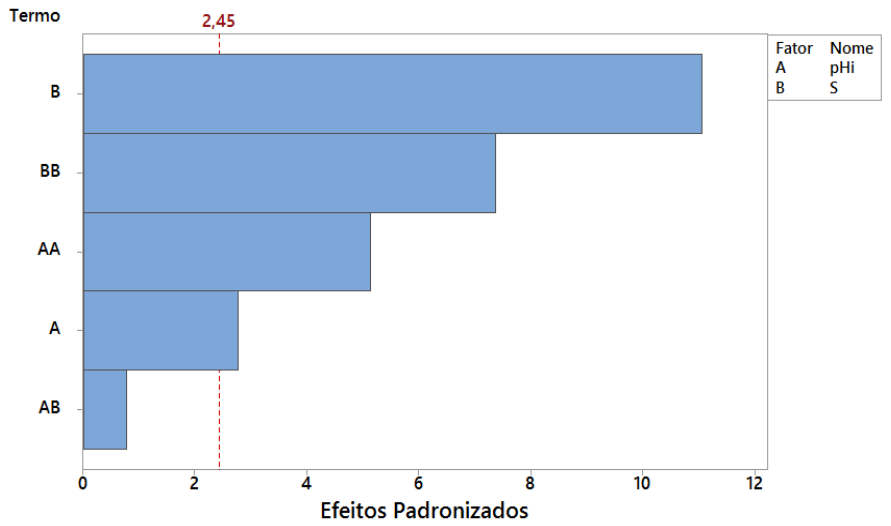

Figura 5 - Gráfico de pareto dos Efeitos Padronizados. 
Portanto, o termo da interação $\mathrm{AB}$ foi retirado para ajuste do modelo, representado pela Equação 2. Nota-se, então, que o modelo foi determinado somente pelos termos lineares $\mathrm{pH}$ da água inicial (pHi) e dose de sulfato de alumínio em $\mathrm{mg} \mathrm{L}^{-1}(\mathrm{~S})$, e pelos termos quadráticos $\mathrm{pHi}^{2}$ e $\mathrm{S}^{2}$, sendo $\mathrm{T}$ a remoção percentual de turbidez.

$\mathrm{T}(\%)=-824+231,2 \mathrm{pH}_{\mathrm{i}}+3,417 \mathrm{~S}-15,96 \mathrm{pH}_{\mathrm{i}}{ }^{2}-0,0342 \mathrm{~S}^{2}$

Para avaliar a significância prática dos termos do modelo, fez-se uma análise da grandeza de seus coeficientes codificados (dados não apresentados). Com isso, foi possível perceber que a dose de coagulante $(S)$ exerce uma maior influência sobre a resposta de remoção de turbidez em relação ao pHi. Essa percepção foi a mesma inferida no método Fatorial ao observar o formato alongado das regiões com elevada remoção de turbidez. Além disso, os termos quadráticos $\mathrm{pHi}^{2} \mathrm{e}$ $\mathrm{S}^{2}$ também tiveram maior influência que o termo linear $\mathrm{pHi}$, porém ainda menor que o linear da dose $\mathrm{S}$.

Analisando a Equação 2, observa-se que existem dois termos quadráticos negativos referentes ao $\mathrm{pH}$ inicial e à dose de coagulante, o que indica a possibilidade de otimizar a remoção de turbidez para essas variáveis de entrada. Desse modo, derivando a Equação 2 em relação a cada uma, obtém-se o valor ótimo de pHi de 7,24 e de dose do coagulante de 49,9 $\mathrm{mg} \mathrm{L}^{-1}$, que, teoricamente, corresponde a uma redução de turbidez de 98,60\%, aproximadamente. A Figura 6 apresenta o gráfico de contorno do modelo otimizado, definido por áreas com regiões de diferentes remoções de turbidez e pode-se observar que esse par de valores está localizado no centro da região mais escura dessa figura, a qual representa uma remoção superior a $90 \%$.

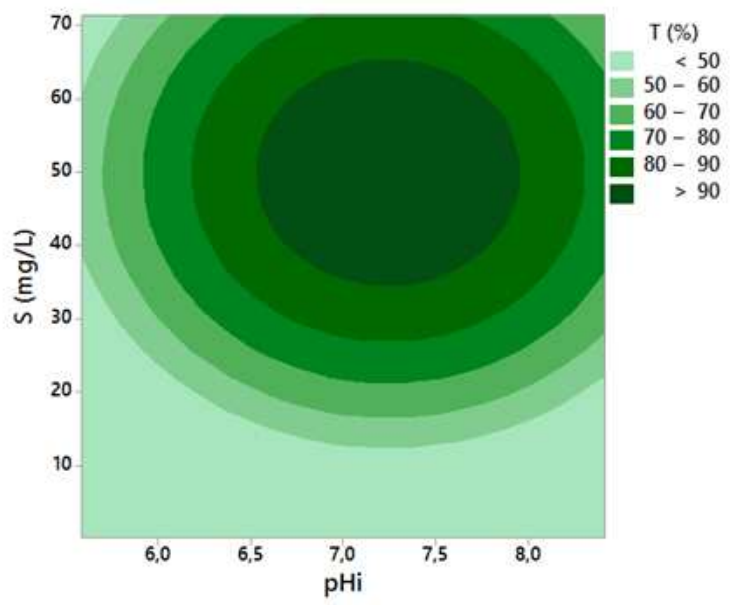

Figura 6 - Gráfico de otimização do DCC para remoção de turbidez.

Pode-se inferir que a otimização do modelo cria uma região acautelada, ou seja, que envolva valores onde pequenas variações de $\mathrm{pH}$ inicial ou dose não afetem, significativamente, na eficácia de remoção da turbidez. Observa-se que essa região ótima está localizada em valores de pHi e S que contemplam regiões de remoção de turbidez dos gráficos das Figuras 3 e 4 que não foram eficientes. Isso pode ser explicado pelo fato de o planejamento realizado utilizar o alfa padrão, maior que 1 , o que deixa os pontos axiais fora dos limites dos pontos cúbicos, como apresentado na Figura 2. Para validação do modelo, seria interessante realizar mais quatro ensaios nos quais os pontos axiais estivessem dentro desses limites, com alfa menor que 1 , de modo a abranger a região da otimização do modelo, como exemplificado na dispersão dos pontos experimentais $\operatorname{com} \alpha=$ 0,7071 da Figura 7.

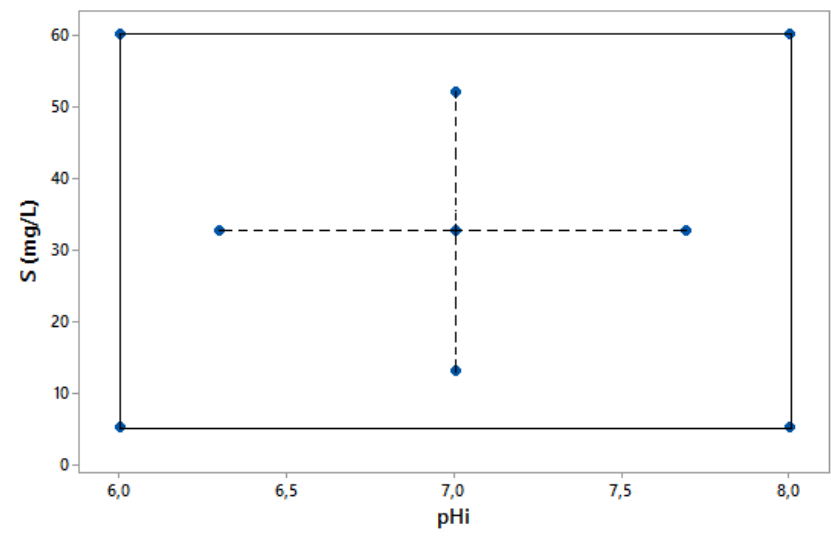

Figura 7 - Gráfico de dispersão dos pontos experimentais da dose de coagulante (S) e do $\mathrm{Ph}$ inicial (pHi), com valor de alfa $=0,7071$.

\subsection{Análise econômica}

Tendo em vista que o tempo necessário para execução de uma corrida de ensaios em um equipamento Jar Test com seis jarros é em média de $114 \mathrm{~min}$, pode-se observar que, para as faixas estudadas, o tempo demandado para os tratamentos utilizando a metodologia de um Fatorial Completo foi cerca de 3,3 vezes maior quando comparado ao Delineamento do Composto Central, conforme apresentado na Tabela 3.

Tabela 3 - Parâmetros e seus respectivos valores utilizados para análise econômica.

\begin{tabular}{lcc}
\hline \hline Parâmetros & Fatorial & MSR \\
Faixa pHi & $6-8$ & $6-8$ \\
Faixa dose / mg L & $5-60$ & $5-60$ \\
Ensaios necessários & 60 & 13 \\
Corridas necessárias & 10 & 3 \\
Tempo por ensaio /min & 114 & 114 \\
Tempo total / min & 1140 & 342 \\
\hline
\end{tabular}

Considerando que a jornada de trabalho de um operador de uma estação de tratamento de água é, em média, 8 horas por dia, pode-se inferir que seriam necessários 2 dias e 3 horas para a execução dos ensaios para o Fatorial Completo e 5,7 horas para o DCC. Essa diferença seria ainda maior caso se desejasse, por exemplo, dobrar a quantidade de níveis definidos para a mesma faixa de dosagem de coagulante, resultando um total de 120 ensaios. Nesse caso, o operador precisaria de 4 dias e 6 horas para executar os tratamentos pelo Fatorial Completo e não haveria alteração para o DCC.

Utilizando como base para cálculo um salário mensal de operador igual a $\mathrm{R} \$ 3.000,00$, tem-se que para a realização dos ensaios seriam gastos $\mathrm{R} \$ 285,00$ e $\mathrm{R} \$ 85,50$, para o Fatorial e DCC, respectivamente. $\mathrm{O}$ custo relativo à demanda do coagulante sulfato de alumínio não foi incluído na análise, pois a diferença dos valores entre os métodos não foi significativa.

Os resultados configuram um quadro de economia de $70 \%$ de tempo e preço para ETA's que optem pela utilização do DCC, o que reduz consideravelmente despesas para execução dos ensaios necessários. 


\section{CONCLUSÕES}

Foram avaliados modelos estatísticos para a análise de remoção de turbidez da água originada do Córrego dos Araújos após as etapas de floculação/coagulação e sedimentação. Dessa forma, foi possível avaliar comparativamente os gráficos de contorno dos dados reais para o método do Fatorial Completo e do DCC. A partir da visualização das regiões de remoção percentual de turbidez com diferentes valores de $\mathrm{pH}$ inicial e dose de coagulante, foi possível observar a ocorrência, em ambos os gráficos, de regiões com eficiência superior a $80 \%$.

O DCC apresenta um número menor de ensaios, que diminui significativamente o tempo de realização dos experimentos e, consequentemente, o custo. Infere-se que o DCC é uma boa alternativa para experimentos que envolvam mais de uma variável de interesse com intuito de avaliar uma ou mais respostas. Além disso, pode ser um bom auxílio quando se deseja construir uma nova ETA, otimizar o tratamento ou, até mesmo, testar a eficiência de diferentes coagulantes.

Foi possível também, pelo DCC, observar que a dosagem de coagulante teve uma maior influência na variável de saída, em relação ao pHi. Definiu uma região boa de remoção de turbidez que extrapola os limites de pHi e dosagem estabelecidos, sendo um ponto positivo em relação ao Fatorial que não teve ensaios além dessas faixas e, por consequência, negligenciou uma boa região de resposta.

Entretanto, além de não apresentar algumas regiões que o Fatorial foi capaz de contemplar, o DCC também requer uma capacitação de pessoal para planejamento dos ensaios e análise dos resultados, a partir de um software estatístico.

A equação encontrada a partir do Delineamento Composto Central foi otimizada para remoção de turbidez, o que gerou uma região segura onde poucas variações nos parâmetros de entrada não resultariam em remoções, significativamente, baixas. Entretanto, faz-se necessária a validação do modelo, pois essa região ótima não é coincidente com as que surgiram pelos gráficos de contorno dos dados reais.

É importante salientar que, mesmo os ensaios tendo apresentado combinações cuja remoção de turbidez foi elevada, faz-se extremamente necessário uma análise microbiológica e organoléptica, bem como avaliar a presença de cianotoxinas, de modo a atender as exigências para o abastecimento público verificando a eficiência do tratamento convencional, sendo um assunto relevante para trabalhos futuros, bem como analisar a necessidade e/ou eficiência da implementação de prétratamentos.

\section{A GR A D E C I M E N T O S}

A todos os funcionários da Estação de Tratamento de Água (ETA-UFV) e aos professores Antônio Galvão do Nascimento e Alisson Borges, por toda ajuda e incentivo.

R E F E R E N C I A S

AMIRTHARAJH, A. Tecnologias para tratamento de águas de abastecimento - The mechanisms of coagulation. 1989.

AREND, D. N. Minitab 17 - Statistical Software; State College, PA: Minitab, Inc, 2010.
BARTIKO, D.; JULIO, M. D. Construção e emprego de diagramas de coagulação como ferramenta para o monitoramento contínuo da floculação em águas de abastecimento. Revista Ambiente \& Água, vol. 10, n. 1, p. 71-81, 2015.

BEZERRA, M. A.; SANTELLI, R. E.; OLIVEIRA, E. P.; VILLAR, L. S.; ESCALEIRA, L. A. Response surface methodology (RSM) as a tool for optimization in analytical chemistry. Talanta, v. 76, n. 5, 2008.

BRASIL. IBGE. Pesquisa Nacional de Saneamento Básico, 2008. Disponível em: <https://biblioteca.ibge.gov.br>, acesso em 27 de maio, 2018.

BRASIL. Ministério da Saúde; PRC n 5, 28 de set. 2017, anexo XX, Diário Oficial da União, Brasília, DF, 2017. Disponível em: <http://bvsms.saude.gov.br>, acesso em 20 de maio, 2018.

COMBATT, M. P. M.; MENDONÇA, R. C. S.; VALENTE, G. F. S; SILVA, C. M.; Validação do processo de eletrocoagulação e avaliação da eletrodissolução de eletrodos no tratamento de efluentes de abatedouros de aves, Revista Química Nova, v. 40, n.4, 447-453, fev. 2017.

DI BERNARDO, L.; DANTAS, A. D. B.; Métodos e técnicas de tratamento de água. $2^{\mathrm{a}}$ ed., v. 1, São Carlos, Brasil: RiMa. 2005.

DI BERNARDO, L.; DI BERNARDO, A.; VOLTAN, P. L. Tratabilidade de água e dos resíduos gerados em estações de tratamento de água. [S.1.]: LdiBe, 2011.

DUAN, J., GREGORY, J. Coagulation by hydrolysing metal salts, Adv. Colloid Interface Sci., 2003.

EATON, A. D. Standard Methods for the Examination of Water and Wastewater, $23^{\text {th }}$ ed., 2017.

FERRARI, T. N.; DE JULIO, M.; DE JULIO, T. S.; SOUZA JÚNIOR, W. C. Estudos de Tratabilidade das Águas do Rio Paraíba do Sul que Abastecem o Município de São José dos Campos/SP. Revista AIDIS de Ingeniería y Ciencias Ambientales, v.5, n. 2, p. 45-58, ago. 2012.

GOMES, F.; SOUSA, M. Subsídios para formulação de políticas de gestão de recursos hídricos na UFV, campus Viçosa, Universidade Federal de Viçosa. 2015. 67f. Monografia (Curso de Engenharia Ambiental) Universidade Federal de Viçosa, Viçosa, MG, 2015.

INSTITUTO MINEIRO DE GESTÃO DAS ÁGUAS (IGAM). Disponível em: <www.igam.mg.gov.br>, acesso em 07 de julho, 2018.

LIBANIO, M.; Fundamentos de Qualidade e Tratamento de Água. $3^{\text {a }}$ ed., Campinas, São Paulo: Átomo, 2010.

MORAIS, A. A. Avaliação de técnicas convencionais de tratamento de água para consumo humano na remoção de cianobactérias e cianotoxinas e processos oxidativos para remoção de microcistinas. 2012. 159f. Dissertação (Doutorado em Engenharia Civil) - Universidade Federal de Viçosa, Viçosa, MG, 2012.

OLIVEIRA, D. C. Aplicação da análise de perigos e pontos críticos de controle no tratamento de água para consumo humano. 2009. 114f. Dissertação (Mestrado em Hidráulica e Saneamento), Universidade Federal de Viçosa, Viçosa, MG, 2009.

PAVANELLI, G. Eficiência de diferentes tipos de coagulantes na coagulação, floculação e sedimentação de água com cor ou turbidez elevada. 2001. 216f. Dissertação (Mestrado em Hidráulica e Saneamento) - Escola de 
Engenharia de São Carlos, Universidade de São Paulo, SMITH, V. H.; SCHINDLER, D. W.; Eutrophication science: São Carlos, SP, 2001.

RIBEIRO JÚNIOR, J. I. Métodos Estatísticos Aplicados à where do we go from here? Trends in Ecology and

Melhoria da Qualidade. Viçosa: UFV, 2012. Evolution, v. 24, n. 4, 2009. 